\title{
Open Eye
}

National Cancer Institute

\section{Source}

National Cancer Institute. Open Eye. NCI Thesaurus. Code C124567.

A finding in which the eye appears open at birth, either due to the congenital absence of eyelids or incomplete fusion of the eyelid. 